\title{
Pain Alleviation and Opioid Weaning in an 80-Year-Old with Chronic Foot Pain Following Injection Therapy with Perineural Dexmedetomidine and Dexamethasone
}

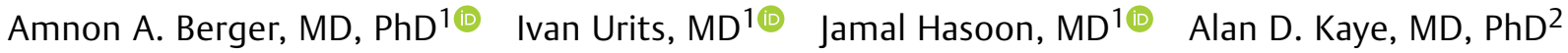 \\ Omar Viswanath, $\mathrm{MD}^{3,4,5}$ Jonathan Eskander, MD, MBA ${ }^{6}$
}

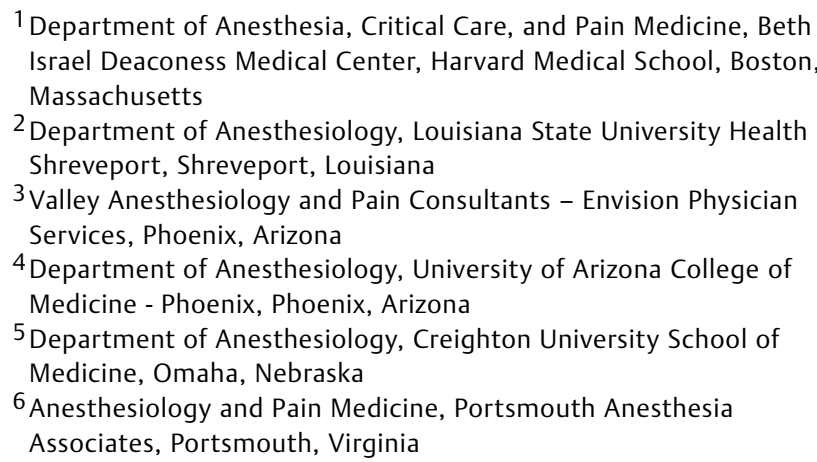

Address for correspondence Amnon A. Berger, MD, PhD, Department of Anesthesia, Critical Care, and Pain Medicine, Beth Israel Deaconess Medical Center, Harvard Medical School, 330 Brookline Ave, Boston, MA, 02215 (e-mail: aberger1@bidmc.harvard.edu).

Surg J 2021;7:e1-e2.

\begin{abstract}
Opiates are routinely used for chronic pain patients, and up to $44 \%$ of them will have a prescription for an opiate medication for pain alleviation. However, of the 76 million adults prescribed opiates for pain management, about $12 \%$ report misuse, and a large number of these may find themselves addicted to opioid medications. Opioid addiction is an ongoing epidemic, costing many lives. Withdrawal is very difficult. This requires providers to

Keywords

- pain therapy

- chronic pain

- opioid withdrawal

- Dex-Dex consider alternative analgesic plans and minimize opiate use. Here we report the use of a dexamethasone-dexmedetomidine combination for a regional nerve block in an elderly woman chronically treated with opiate medications who had previously failed opiate weaning. Following her nerve block, she was able to completely wean off of opioids and continues having good pain control with an opioid-free regimen.
\end{abstract}

Morphine is a potent analgesic and is often treated as the opiate prototype. Up to $44 \%$ of chronic pain patients are prescribed an opiate for pain alleviation. ${ }^{1}$ Through reward pathway activation, morphine treats pain, but there is also a risk of opioid addiction. ${ }^{2}$ It is estimated that of the 76 million adults were prescribed opioid drugs in 2016 to 2017, and 12\% reported opioid misuse, often the first step leading to addiction. ${ }^{3}$ In 2017, United States recorded 47,506 opioid deaths (13.21 per 100,000, up from 2.20 in 2004). ${ }^{4}$ Withdrawal is often difficult, and relapse rates are high.

received

March 24, 2020

accepted after revision

September 22, 2020
DOI https://doi.org/

$10.1055 / \mathrm{s}-0040-1722176$.

ISSN 2378-5128.
We briefly report the case of an 80-year-old patient who was previously diagnosed with chronic foot pain and treated with opiate medication for longer than a decade. She has had numerous previous foot and ankle surgeries, including arthroscopy, neuroma excision, and Achilles tendon lengthening; however, she continued to have chronic foot pain, mostly affecting the medial and dorsal aspects of her foot, which she described as a constant burning sensation with intermitted superimposed sharp pain. The pain was neuropathic in nature and $7 / 10$ in severity on a 10 -

(c) 2021. The Author(s).

This is an open access article published by Thieme under the terms of the Creative Commons Attribution License, permitting unrestricted use, distribution, and reproduction so long as the original work is properly cited. (https://creativecommons.org/licenses/by/4.0/)

Thieme Medical Publishers, Inc., 333 Seventh Avenue, 18th Floor, New York, NY 10001, USA 
point scale, with exacerbation up to $10 / 10$ with touch and activity. She was managed with increased doses of opiates, and she has been taking $80 \mathrm{mg}$ of morphine equivalents for the 10 years preceding her presentation. Previous attempts of weaning down opioid doses failed due to the persistence of pain.

Most recently, this patient enrolled in a detoxification program targeting opioid detoxification within 1 to 2 weeks. This program uses a combination of weak opioids, benzodiazepines, promethazine, and possibly buprenorphine-naloxone (Saboxone), during outpatient treatment. Given the previous failure of detoxification and refractory pain, the patient underwent injection therapy to address her pain. Previous cases demonstrate prolonged nerve blockade using the dexmedetomidine and dexamethasone (Dex-Dex) combo. ${ }^{5-7}$ She was given an adductor canal block, using a mixture of $0.2 \%$ ropivacaine with $5 \mathrm{mg}$ preservative-free dexamethasone and 25 mcg of dexmedetomidine, alongside a popliteal block with local anesthetic and dexamethasone, to achieve full coverage. We decided to not use the Dex-Dex combo for the popliteal fossa block, given its ability to produce an unpredictably long sensory blockade along the common peroneal distribution.

Following this procedure, the patient has had complete pain relief for approximately 1 week and was able to wean off of opioid use entirely, from which she continues to abstain. Her overall pain was minimal at baseline and up to 1 to $2 / 10$, with exacerbation during this 1 week follow-up. Her pain is now successfully managed using a combination of medical marijuana and cannabidiol (CBD) oil, which she uses about once weekly.

The actual mechanism of the Dex-Dex combination is unknown. Dexmedetomidine is a $\alpha 2$ receptor agonist. which is known to provide analgesia through supraspinal, spinal, and peripheral actions. Local action is known to prolong the effect of local anesthetics, and the mechanism is most probably via hyperpolarization-activated cation currents. Dexamethasone likely prolongs the effect of local anesthetics through its antiinflammatory properties, most probably through the inhibition of prostaglandin formation and release of endorphins. Previous data found a synergistic effect of the combination, with a yet-to-be-discovered mechanism or action. ${ }^{5-7}$

This case demonstrates an example of including injection therapy as an adjunct for opioid detoxification to address chronic pain in long-term opioid users. By addressing the pain component in this patient, we were able to potentiate medical therapy addressing the other aspects of withdrawal and detoxification and allow for a safe and continued opioid rehabilitation. This multimodal approach was successful in achieving long-term detoxification in a chronic opioid user. With the growing opioid misuse epidemic, there is a need for continually evolving approaches to facilitate detoxification and injection therapy is an important adjunct.

\section{Disclosures}

The authors have nothing to disclose.

\section{Funding}

No external funding source was used for this publication.

Conflict of Interest

None declared.

\section{References}

1 Clark JD. Chronic pain prevalence and analgesic prescribing in a general medical population. J Pain Symptom Manage 2002;23 (02):131-137

2 Kim J, Ham S, Hong H, Moon C, Im HI. Brain reward circuits in morphine addiction. Mol Cells 2016;39(09):645-653

3 Becker WC, Starrels JL. Prescription drug misuse: epidemiology, prevention, identification, and management. https://www. uptodate.com/contents/prescription-drug-misuse-epidemiologyprevention-identification-and-management?search=pharmacotherapy-for-alcohol-use-disorder\&topicRef=16750\&source=see_link. Accessed March 16, 2020

4 Olfson M, Rossen LM, Wall MM, Houry D, Blanco C. Trends in intentional and unintentional opioid overdose deaths in the United States, 2000-2017. JAMA 2019;322(23):2340-2342

5 Moeen SM, Ramadan IK, Elkady HA. Dexamethasone and dexmedetomidine as an adjuvant to intraarticular bupivacaine for postoperative pain relief in knee arthroscopic surgery: a randomized trial. Pain Physician 2017;20(07):671-680

6 Herman J, Urits I, Hasoon J, et al. Synergistic effect of local dexamethasone and dexmedetomidine (Dex-Dex) in extending the analgesic effect of a transversus abdominis plane block prior to inguinal hernia repair. J Clin Anesth 2020;62:109703

7 Herman J, Urits I, Urman RD, Kaye AD, Viswanath O, Eskander JP. Synergistic effect of perineural dexamethasone and dexmedetomidine (Dex-Dex) in extending the analgesic duration of a transversus abdominis plane block. J Clin Anesth 2020;63:109750 